\title{
A Preliminary Study of CT Texture Analysis for Characterizing Epithelial Tumors of the Parotid Gland
}

This article was published in the following Dove Press journal: Cancer Management and Research

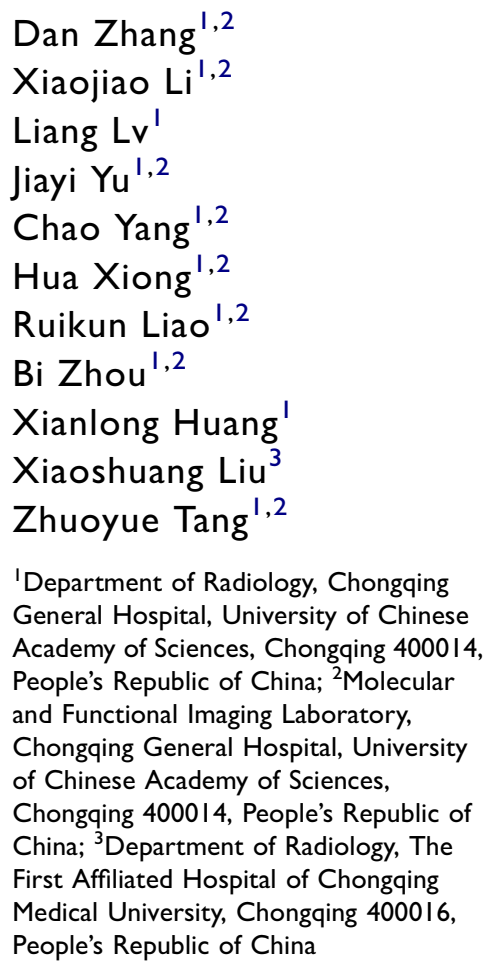

Correspondence: Zhuoyue Tang Department of Radiology, Chongqing General Hospital, University of Chinese Academy of Sciences, Chongqing 4000I4, People's Republic of China

Email zhuoyue_tang@ucas.ac.cn
Objective: The aim of this study was to explore and validate the diagnostic performance of whole-volume CT texture features in differentiating the common benign and malignant epithelial tumors of the parotid gland.

Materials and Methods: Contrast-enhanced CT images of 83 patients with common benign and malignant epithelial tumors of the parotid gland confirmed by histopathology were retrospectively analyzed, including 50 patients with pleomorphic adenoma (PA) and 33 patients with malignant epithelial tumors. Quantitative texture features of tumors were extracted from CT images of arterial phase. The diagnostic performance of texture features was evaluated via receiver operating characteristic (ROC) curve and area under ROC curve (AUC). The specificity and sensitivity were respectively discussed by the maximum Youden's index.

Results: All the texture features were subject to normal distribution and homoscedasticity. Energy, mean, correlation, and sum entropy of epithelial malignancy group were significantly higher than those of PA group $(\mathrm{P}<0.05)$. There were no statistically significant differences between PA group and epithelial malignancy group in uniformity, entropy, skewness, kurtosis, contrast, and difference entropy $(\mathrm{P}>0.05)$. The AUC of each texture feature and joint diagnostic model was 0.887 (energy), 0.734 (mean), 0.739 (correlation), 0.623 (sum entropy), 0.888 (energy-mean), 0.883 (energy-correlation), 0.784 (mean-correlation). The diagnostic efficiency of energy-mean was the best. Based on the maximum Youden's index, the specificity of energy-correlation was the highest (97\%) and the sensitivity of energy was the highest (97\%).

Conclusion: Energy, mean, correlation, and sum entropy can be the effective quantitative texture features to differentiate the benign and malignant epithelial tumors of the parotid gland. With higher AUC, energy and energy-mean are superior to other indexes or joint diagnostic models in differentiating the benign and malignant epithelial tumors of the parotid gland. CT texture analysis can be used as a noninvasive and valuable means of preoperative assessment of parotid epithelial tumors without additional cost to the patients.

Keywords: texture analysis, epithelial tumors, parotid gland

\section{Introduction}

The diversity of histological and clinical manifestations is a significant feature of parotid tumors. The histological diversity combined with the rarity of these tumors makes it difficult to provide available noninvasive diagnostic recommendations for each individual histological subtype of parotid tumors. ${ }^{1}$ 
Generally, parotid tumors are present in two forms: a well-defined and mobile lump or a lump with significant accompanying symptoms, like pain, rapid growth, facial paralysis or metastasis. The former features are indications of benign lesions, while the latter features are indications of malignancy. However, quite a few malignancies have an indolent nature and may be clinically indistinguishable from benign tumors. As indolent lesions may masquerade as benign tumors, the definitive histology sometimes may not be available until after surgical resection. At present, partial parotidectomy or superficial parotidectomy is usually applied for benign tumors and partial superficial parotidectomy (PSP) is the most common surgical procedure for PA, while total parotidectomy with or without facial nerve removal is applied to malignant tumors., ${ }^{2,3}$ Consequently, preoperative diagnosis of parotid gland tumors is of great importance in view of optimizing the individualized surgical plan. Differentiation based on clinical symptoms is difficult because of the obvious overlap among different tumors.

Ultrasound is useful in the diagnosis of parotid tumors, but it is difficult to detect masses located in the deep lobe and the results closely depend on the experience and skill level of the operator. ${ }^{4}$ Magnetic resonance imaging (MRI) seems to have the highest potential to determine different histological subtypes of parotid tumors noninvasively. Nevertheless, its disadvantages are limited availability, high cost and the long time needed. Patients with certain pacemakers or implanted metals cannot be examined with MRI. ${ }^{5}$ Fine-needle aspiration cytology (FNAC) is a minimally invasive procedure usually used in preoperative diagnosis. However, its result is not always conclusive due to insufficient specimens. ${ }^{6}$ Open biopsy is not encouraged in apparently benign tumors as it carries a theoretical risk of seeding, but it sometimes has a role in the frankly malignant tumors, especially when radical surgery is being contemplated. Therefore, it is urgently necessary to find an effective and relatively safe method to characterize parotid tumors before operation.

Some recent studies have demonstrated the potential value of texture features, which are significantly associated with the pathology of tumors and the prognosis of patients. $^{7,8}$ Texture features can be extracted from many kinds of medical images and provide quantitative information we need. ${ }^{9}$ A great deal of quantitative information is contained in medical images, which is far beyond what is currently used for routine interpretation. Consequently, more and more interest is focused on the evaluation of tumors on medical images though a variety of advanced software with the purpose of acquiring additional, clinically relevant information, such as texture analysis. Texture analysis has been used to predict several clinical issues, like tumor heterogeneity, patient prognosis, response to therapy, and so on. ${ }^{10,12}$ In this study, texture features were extracted from contrast-enhanced CT images for characterizing epithelial tumors of the parotid gland.

As far as we know, very few data on the texture features derived from malignant epithelial tumors and PAs, which is the most common benign epithelial tumor, have already been analyzed. Consequently, this study focused on differentiating benign from malignant epithelial neoplasms of the parotid gland by texture analysis.

\section{Materials and Methods Patient Selection}

This retrospective study was approved by the local Ethics Committee, and all patients data were maintained with confidentiality, in accordance with the Declaration of Helsinki. Between January 2016 and December 2017, patients with PA or malignant epithelial tumors who underwent surgery were eligible and identified from the institution's database. The clinical information of patients in the PA group and malignant epithelial tumor group is shown in Table 1.

The inclusion criteria were as follows: (1) PA or malignant epithelial tumors of the parotid gland confirmed by postoperative pathology; (2) complete CT contrastenhanced images of neck obtained within two weeks prior to surgery; and (3) maximum diameter of lesions $\geq 2.0 \mathrm{~cm}$ in the cross section. The exclusion criteria were as follows: (1) CT images with obvious artifacts, such as false teeth artifacts, motion artifacts, etc.; and (2) lesions with scarcely solid components which are difficult for texture analysis. As a result, a total of 149 patients were identified, and 66 patients were excluded (Figure 1). The final study population was comprised of 83 patients.

\section{Image Acquisition}

CT scans were performed using 64/128-multidetector scanners (LightSpeed VCT; GE Healthcare, Waukesha, WI, USA) with the parameters as follows: tube voltage, $120 \mathrm{kVp}$; tube current, $150 \mathrm{~mA}$; section thickness, $5 \mathrm{~mm}$; section interval, $5 \mathrm{~mm}$. The scanning ranged from the base of the skull to the inlet of thorax. The enhanced images were obtained after intravenous injection of $80-100 \mathrm{~mL}$ of nonionic iodinated 
Table I Baseline Patient Characteristics in PA Group and Malignant Epithelial Tumor Group

\begin{tabular}{|c|c|c|c|c|}
\hline Characteristics & & Pleomorphic Adenoma $(\mathrm{N}=50)$ & Malignant Tumor $(\mathrm{N}=33)$ & $P$ value \\
\hline $\mathrm{Age}^{\mathrm{a}}$ (year) & & $47.820 \pm 12.605$ & $47.424 \pm 13.730$ & 0.893 \\
\hline Gender $^{\mathrm{b}}$ & $\begin{array}{l}\text { Male } \\
\text { Female }\end{array}$ & $\begin{array}{l}13(26 \%) \\
37(74 \%)\end{array}$ & $\begin{array}{l}24(72.7 \%) \\
9(27.3 \%)\end{array}$ & $<0.01$ \\
\hline Clinical presentation ${ }^{\mathrm{b}}$ & $\begin{array}{l}(+) \\
(-)\end{array}$ & $\begin{array}{l}I(2 \%) \\
49(98 \%)\end{array}$ & $\begin{array}{l}7(21.2 \%) \\
26(78.8 \%)\end{array}$ & 0.006 \\
\hline Disease duration ${ }^{c}$ (month) & & $12.000(4.000,39.000)$ & $8.000(4.500,21.000)$ & 0.093 \\
\hline Lymphadenopathy & & $0(0 \%)$ & $4(12.1 \%)$ & 0.022 \\
\hline
\end{tabular}

Notes: ${ }^{a}$ Data: Mean \pm SD. ${ }^{b}$ Data: No. (percentage). ' Data: M. (QI, Q3).

Abbreviations: PA, pleomorphic adenoma; $\mathrm{N}$, number.

contrast material $(320 \mathrm{mg} / \mathrm{mL}$; Iopamidol, Shanghai Bracco Sine Pharmaceutical Co., Ltd., Shanghai, China) at an injection rate of $3.0 \mathrm{~mL} / \mathrm{s}$, followed by $50 \mathrm{~mL}$ of saline solution via a power injector. The contrast-enhanced $\mathrm{CT}$ images were obtained at 35 and 120 seconds after contrast material injection in arterial phase and balanced phase, respectively.

\section{CT Texture Features}

All CT images of arterial phase were stored in Digital Imaging and Communications in Medicine (DICOM) format and uploaded to ITK-SNAP software for threedimensional manual segmentation of the region of interest (ROI). ROI of each case was manually drawn by a head and

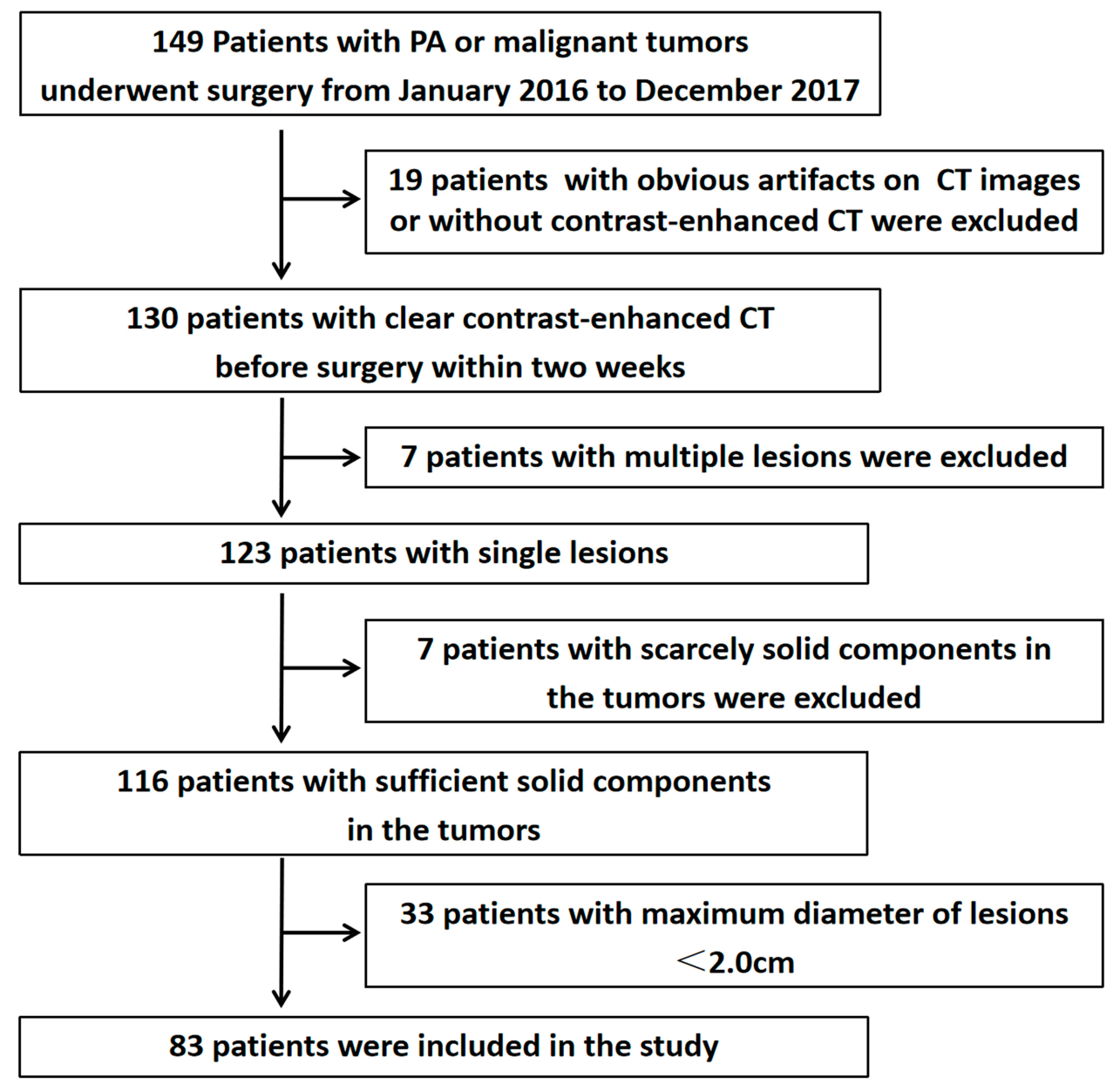

Figure I Recruitment pathway of patients in this study. 
neck radiologist who did not have any knowledge about the clinical information of patients, and then the segmentation was checked by a senior radiologist. Areas of tumor heterogeneity, including cystic change or necrosis, were not excluded, for the information captured with texture analysis could potentially contribute to tumor discrimination and classification. Meanwhile, the delineation should be as close as possible to the edge of the lesion by $1-2 \mathrm{~mm}$, so as to avoid drawing too much parotid parenchyma or posterior mandibular vein into the lesion, which may lead to a misunderstanding of the internal structure of the tumor and affect the accuracy of texture analysis. An in-house software, Matlab2017b (Mathworks, Natick, MA, USA), was used to extract the texture parameters automatically. Ten frequently-used texture features obtained from the graylevel histogram and gray level co-occurrence matrix were included in the study, namely uniformity (uniformity of gray-level distribution), mean (average pixel value), energy (homogeneity in an image), skewness (asymmetry of the histogram), and kurtosis (flatness of the histogram), contrast (difference moment of the matrix), correlation (measurement of gray-tone linear dependencies), entropy (irregularity of gray-level distribution), difference entropy, and sum entropy. ${ }^{13}$ An overview of the texture features extraction process and analysis is shown in Figure 2.

\section{Data Analysis}

Data management and statistical analysis were conducted by IBM SPSS Statistics package (version 25, SPSS Inc., Chicago, IL, USA). Kolmogorov-Smirnov test was used for intra-group normality test, and Levene test was used for intragroup variance homogeneity test. Parameters with normal distribution and homogeneity of variances were expressed as mean \pm standard deviation, and independent-samples $t$ test was adopted for data analysis. Parameters that did not satisfy normal distribution and uneven variance were expressed by median and interquartile spacing. The two-independentsamples Mann-Whitney $U$-Test was used for data analysis. Qualitative data were presented as ratios, which were analyzed by chi-square test. The diagnostic performance of single index and pairwise joint model were tested via receiver operating characteristic (ROC) analysis. Cutoff values were established by calculating the maximal Youden index (Youden index $=$ sensitivity + specifcity-1). A two-tailed $\mathrm{P}$ value of less than 0.05 was considered statistically significant.

\section{Results}

\section{Patient Characteristics}

Malignant epithelial tumors of the parotid gland were more common in males, while PAs were more likely in females. Statistically significant difference of gender was found between the two groups $(\mathrm{P}<0.01)$. Meanwhile, significant differences were found in clinical presentation and lymphadenopathy between the two groups $(\mathrm{P}<0.05)$. No statistically significant differences of age and disease duration were found between the two groups $(\mathrm{P}=0.893$ and $\mathrm{P}=0.093$, respectively). All the selected patient characteristics of the two groups are shown in Table 1. The histological subtypes of malignant epithelial tumors included mucoepidermoid carcinoma $(n=10)$, adenoid cystic carcinoma $(n=7)$, salivary duct carcinoma $(n=4)$, squamous cell carcinoma $(n=3)$, acinic cell carcinoma $(n=3)$, epithelial myoepithelial carcinoma $(n=3)$, basal cell adenocarcinoma $(n=2)$, and lymphoepithelial carcinoma $(\mathrm{n}=1)$. Mucoepidermoid carcinoma and adenoid cystic carcinoma were the two most common subtypes. Malignant epithelial tumors were seldom accompanied by clinical presentation and lymphadenopathy (Table 2).

\section{Texture Features}

The energy, mean, correlation, sum entropy of malignant epithelial tumors group were significantly higher than those of PA group (all $\mathrm{P}<0.05$ ). No statistically significant

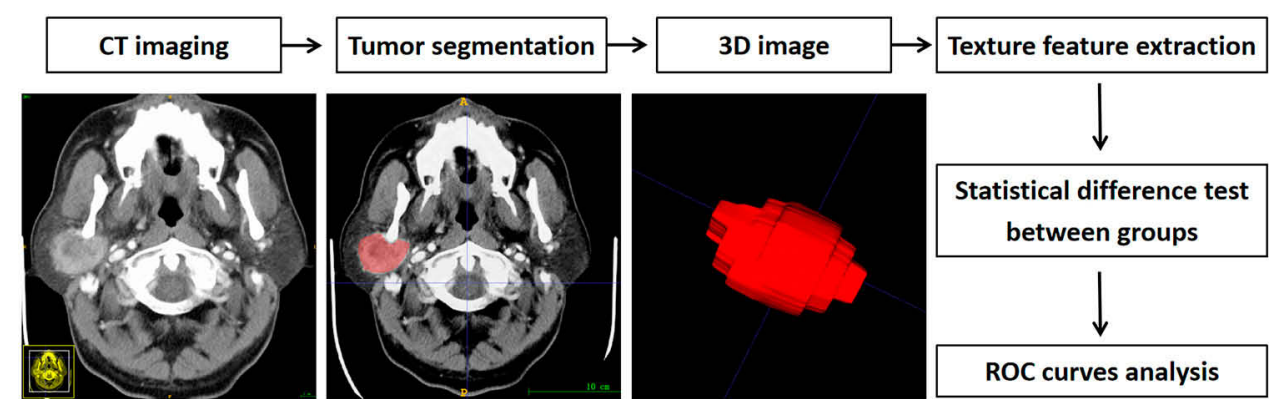

Figure 2 Flowchart illustrating the texture analysis in this study. The original CT images obtained from a patient with mucoepidermoid carcinoma in the right parotid gland. 3D ROls were segmented manually and reconstructed. Texture features were generated automatically and analyzed by ROC curves. 
Table 2 Basic Characteristics of Patients with Parotid Epithelial Malignancy

\begin{tabular}{|l|l|l|l|}
\hline Malignant Tumors & Number & Clinical Presentation & Lymphadenopathy \\
\hline Mucoepidermoid carcinoma & 10 & 2 & $\mathrm{I}$ \\
Adenoid cystic carcinoma & 7 & $\mathrm{I}$ & 0 \\
Salivary duct carcinoma & 4 & $\mathrm{I}$ & 2 \\
Squamous cell carcinoma & 3 & 0 & 0 \\
Acinic cell carcinoma & 3 & $\mathrm{I}$ & 0 \\
Epithelial myoepithelial carcinoma & 3 & $\mathrm{I}$ & 0 \\
Basal cell adenocarcinoma & 2 & 0 & 0 \\
Lymphoepithelial carcinoma & $\mathrm{I}$ & $\mathrm{I}$ & $\mathrm{I}$ \\
\hline
\end{tabular}

differences of uniformity, entropy, skewness, kurtosis, contrast, and difference entropy were found between the two groups (all $\mathrm{P}>0.05$ ) (Table 3 ). The AUC of each texture feature and joint diagnostic model was 0.887 (energy), 0.734 (mean), 0.739 (correlation), 0.623 (sum entropy), 0.888 (energy-mean) , 0.883 (energy-correlation), 0.784 (mean-correlation),respectively. The diagnostic efficiency of energy-mean was the best. According to the maximum Youden's index, the specificity of energy-correlation was the highest (97\%). The sensitivity of energy was the highest (97\%) (Tables 4 and 5). The ROC curve analysis of energy, mean, correlation, and sum entropy is shown in Figure 3, and the ROC curve analysis of energymean, energy-correlation, mean-correlation is shown in Figure 4.

\section{Discussion}

This study aimed to demonstrate whether CT texture analysis could facilitate distinction between parotid PA and malignant epithelial tumors. We calculated the AUC, sensitivity and specificity of different texture features. Our results indicated that $\mathrm{CT}$ texture features might be helpful to diagnose patients with parotid PA and malignant epithelial tumors.

Ultrasound is most commonly used in parotid tumor diagnosis and its usefulness has been improved by elastography gradually. ${ }^{14}$ However, elastography had several disadvantages including high operator dependence in terms of compression and absence of sufficient quantitative information. ${ }^{15}$ FNAC has been proved to be an effective and reliable tool in the preoperative diagnosis of parotid gland tumors. However, the variegated cytomorphology of parotid gland tumors may lead to an error in interpretation and intratumor heterogeneity from a single or limited tumorbiopsy sample can be underestimated. ${ }^{16,17}$ A study showed that sensitivity, specificity, and positive and negative predictive values of FNAC were $84.61 \%, 91.66 \%, 91.6 \%$, and
$85 \%$, respectively, in benign salivary gland lesions, but $84.61 \%, 86.48 \%, 68.75 \%$, and $94.11 \%$, respectively, in malignant salivary gland lesions. There were no differences in sensitivity, but specificity and the positive predictive values were higher in the benign salivary gland lesions while the negative predictive value was higher in malignant lesions. $^{18}$

Previous CT imaging-based research was focused on the morphological characteristics of parotid lesions, such as location, shape, size, margin, density, and enhancement, but results were inconsistent and no diagnostic strategy has been established for the diagnosis of benign and malignant tumors. ${ }^{19}$ MRI has been used to distinguish parotid malignancy from benign tumors. ${ }^{5}$ Nevertheless, its obvious disadvantages include limited availability, high cost and the long waiting and examining time. Certain pacemakers, implanted metals and the like, are the contraindications for MRI examination. Dynamic contrast-enhanced MRI (DCE-MRI) has been proved to be useful for characterizing parotid gland tumors, but the intravenous use of contrast media may lead to some clinical problems. ${ }^{20,21}$ Some previous studies reported that diffusion-weighted imaging (DWI) and its derived apparent diffusion coefficient (ADC) could provide high diagnostic accuracy in characterizing parotid gland tumors, but there was significant overlap in ADC values among different tumors. ${ }^{22-24}$ Therefore, there is still no consensus on the role of DWI in characterizing parotid gland tumors.

Texture features can be derived from CT, MRI, or positron emission tomography (PET) without modification of the acquisition protocols and extra cost to the patients. $^{25,26}$ Recently, texture analysis is principally applied to evaluate the therapeutic effect and prognosis of liver cancer, colorectal cancer, lung cancer, and so on. $^{27-29}$ But it is rarely applied to parotid lesions, except for several reports focusing on the alterations of parotid 


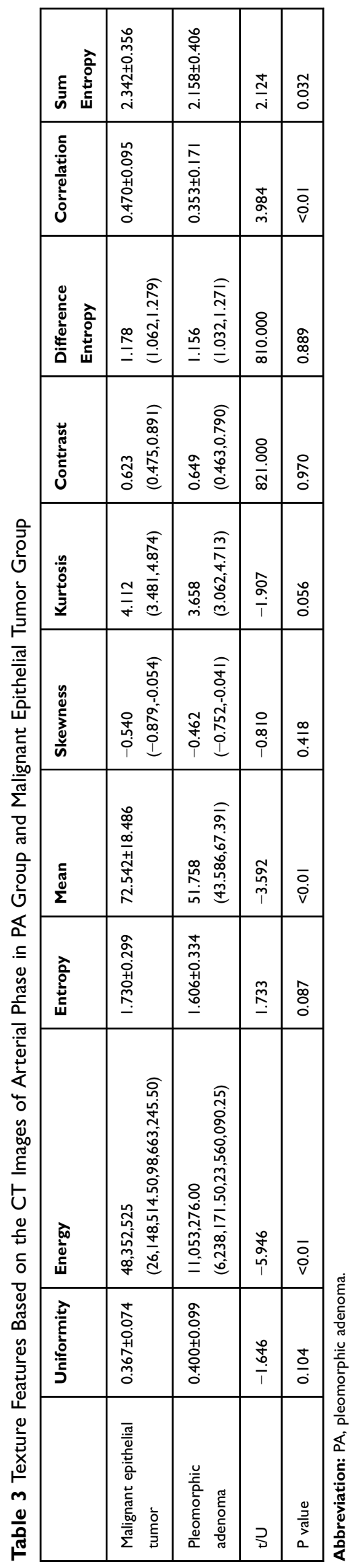

morphology and secretion function induced by radiotherapy. ${ }^{30,31}$ However, to the best of our knowledge, there is a paucity of studies to date about the potential diagnostic value of CT texture analysis in parotid epithelial tumors.

In addition to some features on plain $\mathrm{CT}$, contrastenhanced CT can also reflect some heterogeneous features on tumor blood supply. ${ }^{32}$ Parotid gland tumors are dominated by arterial blood supply, so texture features were extracted from CT images of arterial phase in this study. Because of the heterogeneity of tumors, the lesion often has different texture features in different CT scanning sections. Therefore, the three-dimensional structure instead of single cross-sectional image was studied in this research in order to reduce the random error, although this method may dramatically increase the workload.

We selected 10 frequently-used texture features, among which the energy, mean, correlation, and sum entropy in malignant epithelial tumors were significantly higher than those in PA. The greater energy, mean, correlation, and sum entropy in epithelial malignancy may be related to the greater heterogeneity than benign tumors like PA, although they may appear to be uniformly solid lesions on CT images. These differences in internal density homogeneity are not detected by conventional visual assessments, like CT or MRI.

According to the ROC curve, and comprehensive analysis of AUC, sensitivity, and specificity, it could be concluded that energy and energy-mean were the ideal texture parameter or joint diagnostic model to identify parotid PA and malignant epithelial tumors because of their good diagnosis efficiency, with the AUC of 0.887 and 0.888 , respectively. The sensitivity of energy was the highest (0.970), and the specificity of energy-correlation was the highest (0.970). The energy represents the stability of the lesion texture gray scale change and reflects the gray scale distribution uniformity and texture thickness. The higher the energy is, the more regular and stable the current texture change becomes. In this study, the energy of parotid epithelial malignancy was statistically higher than that of PA. This may be closely related to the histopathological characteristics of PA, such as complicated histological structure and diverse cell types, including glandular epithelium, myoepithelium, mucus, mucoid tissue, and chondroid tissue.

Most parotid gland tumors are benign (80\%), while about $20 \%$ of them are malignant. ${ }^{33}$ A parotid lump is the first sign in most cases. Appearance of malignant tumors may be quite 
Table 4 Diagnostic Performance of CT Texture Features in PA Group and Malignant Epithelial Tumor Group

\begin{tabular}{|l|l|l|l|l|l|l|}
\hline Index & AUC & P value & $\mathbf{9 5 \%} \mathbf{C l}$ & Cutoff Value & Sensitivity & Specificity \\
\hline Energy & 0.887 & $<0.01$ & $0.820-0.955$ & $14,772,788.000$ & 0.970 & 0.620 \\
Mean & 0.734 & $<0.01$ & $0.620-0.848$ & 67.785 & 0.667 & 0.780 \\
Correlation & 0.739 & $<0.01$ & $0.631-0.847$ & 0.422 & 0.788 & 0.700 \\
Sum entropy & 0.623 & 0.059 & $0.502-0.744$ & 1.994 & 0.879 & 0.380 \\
\hline
\end{tabular}

Abbreviation: AUC, area under the ROC curve.

Table 5 Diagnostic Performance of Joint Model of CT Texture Features in PA Group and Malignant Epithelial Tumor Group

\begin{tabular}{|l|l|l|l|l|l|}
\hline Index & AUC & P value & $\mathbf{9 5 \%}$ Cl & Sensitivity & Specificity \\
\hline Energy-mean & 0.888 & $<0.01$ & $0.820-0.955$ & 0.880 & 0.727 \\
Energy-correlation & 0.883 & $<0.01$ & $0.812-0.954$ & 0.660 & 0.970 \\
Mean-correlation & 0.784 & $<0.01$ & $0.687-0.88 \mathrm{I}$ & 0.580 & 0.939 \\
\hline
\end{tabular}

Abbreviation: AUC, area under the ROC curve.

similar to benign tumors, such as growing slowly, mobility and displacement instead of infiltrating neighboring structures. In this study, the average disease duration of PA and malignant epithelial tumors was 12 months, and 8 months, respectively. There is no statistically significant difference of disease duration between the two groups. Only about one third of the malignant parotid tumors present initially with skin infiltration, facial palsy, or lymphatic metastasis as typically clinical signs of malignancy. ${ }^{34}$ In the malignant epithelial tumors group of this study, $21.2 \%$ (7/33) of them appeared the clinical signs, which was roughly in accordance with the previous report. While few patients in the PA group accompanied clinical presentation. It has been reported that the lymph node metastasis rate is approximately $28.2 \%$ in parotid malignancies. ${ }^{35}$ In this study, the lymph node metastasis rate was $12.1 \%$, which was lower than reported. On the one hand,

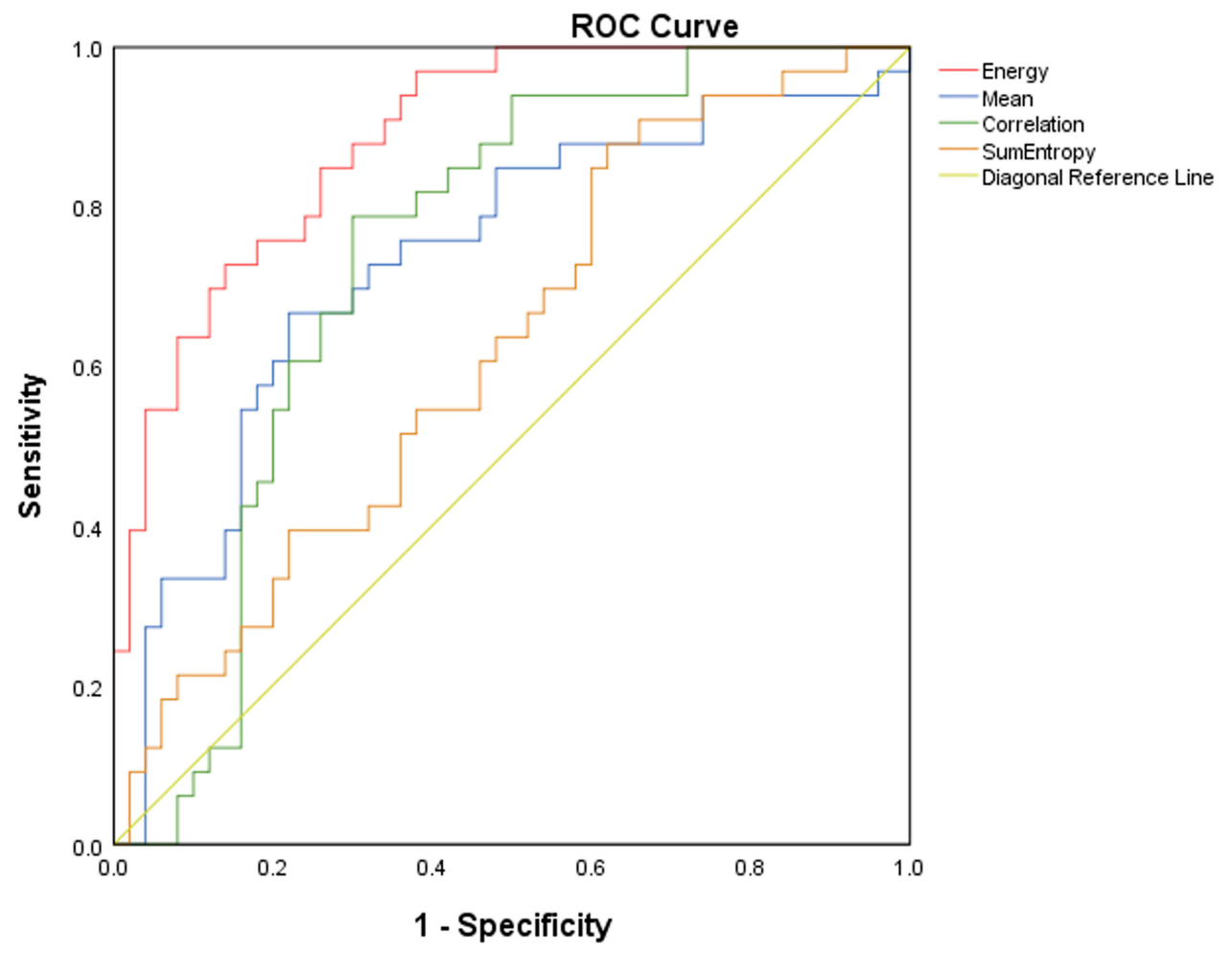

Figure 3 ROC curves for distinguishing PA from malignant epithelial tumor based on CT texture features. 


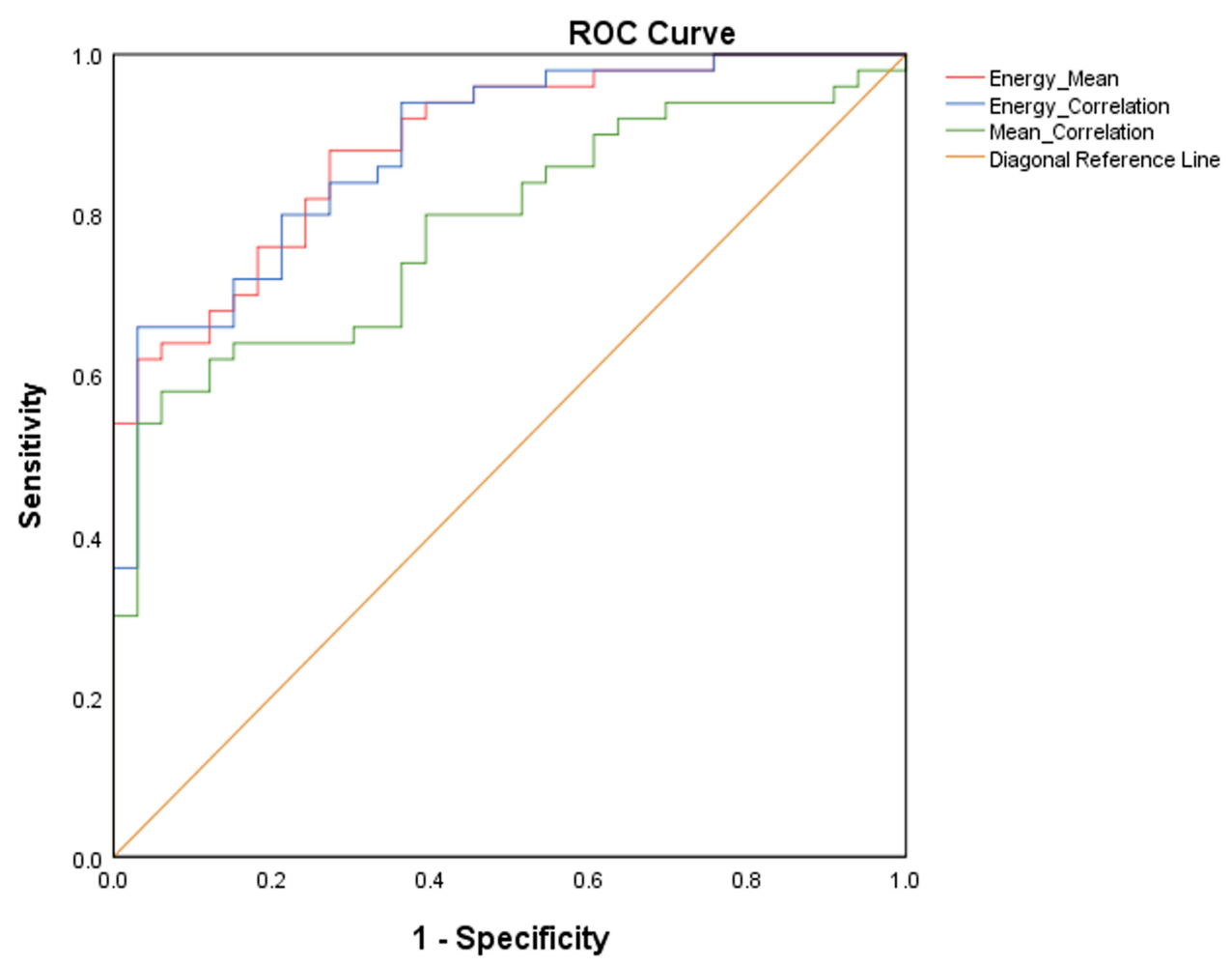

Figure 4 ROC curves for distinguishing PA from malignant epithelial tumor based on joint diagnostic models of CT texture features.

it may be related to the exclusion of the non-epithelial malignancies, like lymphoma, metastasis, and on the other hand it may be due to the small sample size. ${ }^{36}$ Meanwhile, it is necessary to recognize that other non-neoplastic lesions of the parotid gland may also be accompanied by lymphadenopathy, such as kimura's disease and tuberculosis. ${ }^{37}$

At present, PSP is the most common surgical procedure for PAs. While malignant epithelial tumors often need facial nerve rehabilitation management performed together with the oncological surgery. ${ }^{38}$ Moreover, many malignant tumors of the parotid gland need adjuvant radiotherapy. ${ }^{39,40}$ Given the different surgical plans of malignant epithelial tumors and PA, it is hoped that CT texture analysis could improve the diagnostic efficiency and augment the ability for treatment decision-making. In this study, Our findings show that with minimal cost and no additional imaging burden, texture analysis of routine contrast-enhanced CT imaging before surgery may provide useful information for patients with parotid malignant epithelial tumors and PA undergoing different surgical resection. As we know more about the CT texture analysis, it may provide more insights into the different kinds of parotid tumors.

However, the present study still had several limitations. Firstly, the sample size was relatively small. Despite a small sample size, we still found a strong association between texture features and the diagnosis of parotid PA and malignant epithelial tumors. We should investigate the association of a wider range of radiomic features with parotid tumors in a larger sample size. Secondly, since parotid gland tumors are relatively rare diseases in clinical work, and tumor subtypes are characterized by diversity, there is a lack of prospective trials currently. We should conduct prospective studies in the future. Thirdly, cyst adenolymphoma, which is the second most common benign tumors of the parotid gland, was not included in this study, so it will be included in our further study. Finally, there is a lack of understanding on the underlying relationship between texture features and pathology of parotid epithelial tumors, which requires further work.

\section{Conclusions}

In conclusion, we propose and validate the feasibility of CT texture analysis to predict histology of parotid epithelial tumors in this study. Our investigation demonstrates successful application of 3D CT texture analysis for histological classification of the benign and malignant epithelial tumors, which lays a foundation for the future study of texture analysis in other pathology within or outside the head and neck. 


\section{Acknowledgments}

This work was supported by the Medical Research Key Program of the combination of Chongqing National Health Commission and Chongqing Science and Technology Bureau, China (No. 2019ZDXM010); the Basic and Frontier Research Project of Chongqing, China (No. cstc2 016jcyjA0294); the Medical Research Key Program of the National Health and Family Planning Commission of Chongqing, China (No. 20141016 and No. $2016 Z$ DXM026); the Scientific and Technological Innovation Key Program of Chongqing General Hospital, China (No. 2016ZDXM03).

\section{Disclosure}

The authors report no conflicts of interest in this work.

\section{References}

1. Sood S, McGurk M, Vaz F. Management of salivary gland tumours: united Kingdom national multidisciplinary guidelines. J Laryngol Otol. 2016;130(S2):S142-S149. doi:10.1017/s0022215116000566

2. Quer M, Vander Poorten V, Takes RP, et al. Surgical options in benign parotid tumors: a proposal for classification. Eur Arch Otorhinolaryngol. 2017;274(11):3825-3836. doi:10.1007/s00405-017-4650-4

3. Lombardi D, Mcgurk M, Vander Poorten V, et al. Surgical treatment of salivary malignant tumors. Oral Oncol. 2017;65:102-113. doi:10.1016/j.oraloncology.2016.12.007

4. de Ru JA, van Leeuwen MS, van Benthem PP, et al. Do magnetic resonance imaging and ultrasound add anything to the preoperative workup of parotid gland tumors? J Oral Maxillofac Surg. 2007;65:945-952. doi:10.1016/j.joms.2006.04.046

5. Yabuuchi H, Matsuo Y, Kamitani T, et al. Parotid gland tumors: can addition of diffusion-weighted MR imaging to dynamic contrast-enhanced MR imaging improve diagnostic accuracy in characterization? Radiology. 2008;249(3):909-916. doi:10.1148/ radiol.2493072045

6. Witt BL, Schmidt RL. Ultrasound-guided core needle biopsy of salivary gland lesions: a systematic review and meta-analysis. Laryngoscope. 2014;124(3):695-700. doi:10.1002/lary.24339

7. van Dijk LV, Brouwer CL, van der Schaaf A, et al. CT image biomarkers to improve patient-specific prediction of radiation-induced xerostomia and sticky saliva. Radiother Oncol. 2017;122(2):185-191. doi:10.1016/j.radonc.2016.07.007

8. Nardone V, Tini P, Nioche C, et al. Texture analysis of parotid gland as a predictive factor of radiation induced xerostomia: a subset analysis. Radiother Oncol. 2017;122(2):321. doi:10.1016/j.radonc. 2016.09.004

9. Cui Y, Song J, Pollom E, et al. Quantitative analysis of (18) F-fluorodeoxyglucose positron emission tomography identifies novel prognostic imaging biomarkers in locally advanced pancreatic cancer patients treated with stereotactic body radiation therapy. Int $J$ Radiat Oncol Biol Phys. 2016;96(1):102-109. doi:10.1016/j.ijrobp.2016.04.034

10. Chen S, Zhu Y, Liu Z, et al. texture analysis of baseline multiphasic hepatic computed tomography images for prognosis of single hepatocellular carcinoma after hepatectomy: a retrospective pilot study. Eur J Radiol. 2017;90:198-204. doi:10.1016/j.ejrad.2017.02.035

11. Canellas R, Burk KS, Parakh A, et al. Reduction of pancreatic neuroendocrine tumor Grade based on CT features and texture analysis. Am J Roentgenol. 2018;210(2):341-346. doi:10.2214/ AJR.17.18417
12. Liu J, Mao Y, Li Z, et al. Use of texture analysis based on contrast-enhanced MRI to predict treatment response to chemoradiotherapy in nasopharyngeal carcinoma. J Magn Reson Imaging. 2016;44(2):445-455. doi:10.1002/jmri.25156

13. Davnall F, Yip CSP, Ljungqvist G, et al. Assessment of tumor heterogeneity: an emerging imaging tool for clinical practice? Insights Imaging. 2012;3(6):573-589. doi:10.1007/s13244-0120196-6

14. Klintworth N, Mantsopoulos K, Zenk J, et al. Sonoelastography of parotid gland tumours: initial experience and identification of characteristic patterns. Eur Radiol. 2012;22(5):947-956. doi:10.1007/ s00330-011-2344-7

15. Kamaya A, Machtaler S, Safari Sanjani S, et al. New technologies in clinical ultrasound. Semin Roentgenol. 2013;48(3):214-223. doi:10.1053/j.ro.2013.03.009

16. Herman J, Sedlackova Z, Vachutka J, et al. Differential diagnosis of parotid gland tumors: role of shear wave elastography. Biomed Res Int. 2017;2017:9234672. doi:10.1155/2017/9234672

17. Lipinski KA, Barber LJ, Davies MN, et al. Cancer evolution and the limits of predictability in precision cancer medicine. Trends Cancer. 2016;2(1):49-63. doi:10.1016/j.trecan.2015.11.003

18. Singh Nanda KD, Mehta A, Nanda J. Fine-needle aspiration cytology: a reliable tool in the diagnosis of salivary gland lesions. J Oral Pathol Med. 2012;41(1):106-112. doi:10.1111/j.1600-0714.2011. 01069.x

19. Xie D, Zhao W, Liu LD, et al. Distinguishing benign from malignant parotid gland tumours: low-dose multi-phasic CT protocol with 5-minute delay. Eur Radiol. 2011;21(8):1692-1698. doi:10.1007/ s00330-011-2101-y

20. Christe A, Waldherr C, Hallett R, et al. MR imaging of parotid tumors: typical lesion characteristics in MR imaging improve discrimination between benign and malignant disease. Am J Neuroradiol. 2011;32(7):1202-1207. doi:10.3174/ajnr.A2520

21. Kanda T, Fukusato T, Matsuda M, et al. Gadolinium-based contrast agent accumulates in the brain even in subjects without severe renal dysfunction: evaluation of autopsy brain specimens with inductively coupled plasma mass spectroscopy. Radiology. 2015;276(1):228-232. doi:10.1148/radiol.2015142690

22. Tao X, Yang G, Wang P, et al. The value of combining conventional, diffusion-weighted and dynamic contrast-enhanced MR imaging for the diagnosis of parotid gland tumours. Dentomaxillofac Radiol. 2017;46(6):20160434. doi:10.1259/dmfr.20160434

23. Yuan Y, Tang W, Tao X. Parotid gland lesions: separate and combined diagnostic value of conventional MRI, diffusion-weighted imaging and dynamic contrast enhanced MRI. Br J Radiol. 2016;89 (1060):20150912. doi:10.1259/bjr.20150912

24. Habermann CR, Arndt C, Graessner J, et al. Diffusion weighted echo-planar MR imaging of primary parotid gland tumors: is a prediction of different histologic subtypes possible? Am J Neuroradiol. 2009;30(3):591-596. doi:10.3174/ajnr.A1412

25. Koo HJ, Sung YS, Shim WH, et al. Quantitative computed tomography features for predicting tumor recurrence in patients with surgically resected adenocarcinoma of the Lung. PLoS One. 2017;12(1): e0167955. doi:10.1371/journal.pone.0167955

26. Zhai TT, Langendijk JA, van Dijk LV, et al. The prognostic value of CT-based image-biomarkers for head and neck cancer patients treated with definitive (chemo-)radiation. Oral Oncol. 2019;95:178-186. doi:10.1016/j.oraloncology

27. Simpson AL, Doussot A, Creasy JM, et al. Computed tomography image texture: a noninvasive prognostic marker of hepatic recurrence after hepatectomy for metastatic colorectal cancer. Ann Surg Oncol. 2017;24(9):2482-2490. doi:10.1245/s10434-017-5896-1

28. Li Z, Mao Y, Huang W, et al. Texture-based classification of different single liver lesion based on SPAIR T2W MRI images. BMC Med Imaging. 2017;17(1):42. doi:10.1186/s12880-017-0212-X 
29. Pham TD, Watanabe Y, Higuchi M, et al. Texture Analysis and Synthesis of Malignant and Benign Mediastinal Lymph Nodes in Patients with Lung Cancer on Computed Tomography. Sci Rep. 2017;7:43209. doi:10.1038/srep43209

30. Nardone V, Tini P, Nioche C, et al. Texture analysis as a predictor of radiation -induced xerostomia in head and neck patients undergoing IMRT. Radiol Med. 2018;123(6):415-423. doi:10.1007/s11547-0170850-7

31. Wu H, Chen X, Yang X, et al. Early prediction of acute xerostomia during radiation therapy for head and neck cancer based on texture analysis of daily CT. Int J Radiat Oncol Biol Phys. 2018;102 (4):1308-1318. doi:10.1016/j.ijrobp

32. Xu Z, Yong F, Yu T, et al. Different histological subtypes of parotid gland tumors: CT findings and diagnostic strategy. World J Radiol. 2013;5(8):313-320. doi:10.4329/wjr.v5.i8.313

33. Bradley PJ, Mcgurk M. Incidence of salivary gland neoplasms in a defined UK population. Br J Oral Maxillofac Surg. 2013;51 (5):399-403. doi:10.1016/j.bjoms.2012.10.002

34. Guntinas-Lichius O, Wendt TG, Buentzel J, et al. Incidence, treatment, and outcome of parotid carcinoma, 1996-2011: a populationbased study in Thuringia, Germany. J Cancer Res Clin Oncol. 2015;141(9):1679-1688. doi:10.1007/s00432-015-1961-y
35. Kopeć T, Mikaszewski B, Jackowska J, et al. American association of oral and maxillofacial surgeons. J Oral Maxillofac Surg. 2015;73:1397-1402. doi:10.1016/j.joms.2014.12.036

36. Sood S, McGurk M, Vaz F. The Management of salivary gland tumours: united Kingdom national multidisciplinary guidelines. J Laryngol Otol. 2016;130(S2):S142-S149. doi:10.1017/ S0022215116000566

37. Zhang D, Li X, Xiong $\mathrm{H}$, et al. Tuberculosis of the parotid lymph nodes: clinical and imaging features. Infect Drug Resist. 2018;11:1795-1805. doi:10.2147/idr.s164993

38. Volk GF, Pantel M, Guntinas-Lichius O. Modern concepts in facial nerve reconstruction. Head Face Med. 2010;6(1):25. doi:10.1186/ 1746-160X-6-25

39. Lee A, Givi B, Osborn VW, et al. Patterns of care and survival of adjuvant radiation for major salivary adenoid cystic carcinoma. Laryngoscope. 2017;127(9):2057-2062. doi:10.1002/lary.26516

40. Safdieh J, Givi B, Osborn V, et al. Impact of adjuvant radiotherapy for malignant salivary gland tumors. Otolaryngol Head Neck Surg. 2017;157(6):988-994. doi:10.1177/0194599817717661

\section{Publish your work in this journal}

Cancer Management and Research is an international, peer-reviewed open access journal focusing on cancer research and the optimal use of preventative and integrated treatment interventions to achieve improved outcomes, enhanced survival and quality of life for the cancer patient.
The manuscript management system is completely online and includes a very quick and fair peer-review system, which is all easy to use. Visit http://www.dovepress.com/testimonials.php to read real quotes from published authors. 